\title{
Variabilidade espacial em sistema agroflorestal silvibananeiro, no litoral do Rio Grande do Sul com gvSIG
}

\author{
Lúcio de Paula AMARAL ${ }^{1,2 *}$, Regiane Aparecida FERREIRA², Maxiel Gelaim VANZ³, \\ Cristiano MOTTER ${ }^{4}$, André Luiz Rodrigues Gonçalve ${ }^{4,5}$
}

\author{
${ }^{1}$ Departamento de Engenharia Rural, Universidade Federal de Santa Maria, Santa Maria, RS, Brasil. \\ 2Programa de Pós-Graduação em Agricultura de Precisão, Universidade Federal de Santa Maria, Santa Maria, RS, Brasil. \\ ${ }^{3}$ Curso de Engenharia Florestal, Universidade Federal de Santa Maria, Santa Maria, RS, Brasil. \\ ${ }^{4}$ Centro Ecológico, Dom Pedro de Alcântara, RS, Brasil. \\ ${ }_{5}^{5}$ Instituto Federal Catarinense, Santa Rosa do Sul, SC, Brasil. \\ *E-mail: amaralufsm@gmail.com
}

(ORCID: 0000-0001-8542-7078; 0000-0003-0540-6125; 0000-0003-0540-6002; 0000-0001-7373-6375; 0000-0003-3247-4908)

\begin{abstract}
Recebido em 11/09/2020; Aceito em 30/10/2020; Publicado em 12/02/2021.
RESUMO: Os sistemas agroflorestais (SAF) são alternativas importantes para gerar renda com agricultura sustentável, a exemplo dos SAF no Litoral Norte do Rio Grande do Sul. Porém, ainda são poucos os trabalhos que utilizam a abordagem da Agricultura ou Floresta de Precisão, com uso da espacialização de atributos da vegetação dos SAF, sendo este o objetivo desta pesquisa. Foi realizado um inventário, com amostragem aleatória e método da Sexta Árvore de Prodan, num SAF silvibananeiro localizado em Três Cachoeiras (RS), sendo instaladas 33 unidades amostrais (ua) para mensuração dos indivíduos arbóreos com CAP $>15 \mathrm{~cm}$. As ua foram georreferenciadas, calculou-se os parâmetros fitossociológicos, que foram interpolados por ponderação pelo inverso da distância (IDW) no gvSIG, que juntamente com os limites do SAF deram origem a mapas de variabilidade espacial. A principal espécie observada foi palmito juçara (Euterpe edulis Mart. - Família Arecaceae) tendo 75 indivíduos amostrados, com dominância, frequência e densidade relativas de 17, 22,2 e 37\%, respectivamente, e valor de importância de $76,2 \%$. As variáveis que apresentaram variabilidade espacial evidente foram número de espécies, diversidade máxima, índices de diversidade, dominância e equabilidade, número de palmiteiros e cachos de banana verde. Conclui-se que os atributos do SAF silvibananeiro possuem variabilidade espacial e que a metodologia utilizada foi adequada.
\end{abstract}

Palavras-chave: floresta de precisão; manejo florestal de precisão; análise espacial; agrofloresta; agricultura de precisão.

\section{Spatial variability in a silvibanan agroforestry system, on the coast of Rio Grande do Sul with gvSIG}

\begin{abstract}
Agroforestry systems (AS) are important alternatives to generate income from sustainable agriculture, like the AS in the North Coast of Rio Grande do Sul (RS), Brazil. However, there are still few studies that use the precision agriculture or precision forest approach, using the spatialization of attributes of the vegetation AS, this being the objective of this research. An inventory was carried out, with random sampling and the Sixth Prodan Tree Method, in a silvibanan AS located in Três Cachoeiras (RS), with 33 sample units (su) were installed to measure tree individuals with $\mathrm{CBH}>15 \mathrm{~cm}$. The $s u$ were georeferenced, the phytosociological parameters were calculated, which were interpolated by weighting the inverse of the distance (IDW) in the gvSIG, which together with the AS limits gave rise to spatial variability maps. The main species observed was juçara palm (Euterpe edulis Mart. - Family Arecaceae) having 75 individuals sampled, with relative dominance, frequency and density of 17, 22.2 and 37\%, respectively, and importance value of $76.2 \%$. The variables that showed evident spatial variability were number of species, maximum diversity, the index of diversity, dominance and equability, number of palm trees and bunches of green banana. It is concluded that the attributes of the silvibanan AS have spatial variability and that the methodology used was adequate.

Keywords: precision forestry; precision forest management; spatial analysis; agroforestry; precision agriculture.
\end{abstract}

\section{INTRODUÇÃO}

Os Sistemas Agroflorestais (SAF) consistem no cultivo e manejo de várias espécies em um espaço físico ou área de cultivo, ou seja, culturas arbóreas e agrícolas, podendo ocorrer também a criação de animais, manejados em consórcio, com o propósito de se obter uma produção diversificada e constante de alimentos. Os SAF muitas vezes são utilizados para a restauração de áreas degradadas, pois otimizam a produção, melhoram a fertilidade do solo, diminuem riscos de erosão, ataque de pragas e promovem a relação entre plantas e animais. Além disso, os SAF contribuem para a oferta de serviços ambientais, tais como produção de água, biodiversidade, de plantas e animais, e sequestro de carbono (EMBRAPA, 2004). Os SAF mostramse vantajosos comparados aos sistemas convencionais de cultivos, influenciam os atributos físicos e químicos do solo, 
protegendo-os efetivamente contra a erosão, estimulam economicamente a produção, sua sustentabilidade, com base na certificação participativa (MAIA et al., 2006; GARCEZ; MIELITZ NETO, 2008; NUNES; VIVAN, 2011). Os SAF são sistemas inteligentes e diversificados que produzem alimentos, madeiras, entre outros produtos, que podem aumentar a renda do agricultor reduzindo a pressão sobre as florestas nativas, ajudando a conservar e proteger o solo, água, biodiversidade e a fixar carbono, entre outros benefícios (NUNES; VIVAN, 2011; BJÖRKLUND et al., 2012; SILVA, 2013). Esses sistemas são formas de uso ou manejo da terra, nos quais se combinam espécies arbóreas (frutíferas e/ou madeireiras) com cultivos agrícolas e/ou criação de animais, de forma simultânea ou em sequência temporal e que promovem benefícios econômicos e ecológicos, que podem ser considerados serviços ambientais.

Os SAF na região de Três Cachoeiras-RS, próxima a Torres no Litoral Norte do Rio Grande do Sul, são constituídos pela implantação e/ou conversão de bananais convencionais em bananais agroflorestais, também denominados de sistemas silvibananeiros, manejados por agricultores familiares, sob sua ótica cultural, ecológica, econômica, dentre outras, com apoio de organizações não governamentais, a exemplo do Centro Ecológico - Litoral Norte. São consórcios variados de bananeiras (Musa sp), palmito juçara ou palmiteiro (Euterpe edulis Martius), árvores nativas madeiráveis (louro-pardo, cedro, sobragi, canjarana), frutíferas, e uma diversificada variedade de plantas não arbóreas nativas e exóticas, manejadas sob a óptica da agricultura ecológica. Várias espécies florestais são oriundas da regeneração natural (aroeira pimenteira, canelas, alecrim, canjerana, ingás, dentre outras), sendo que algumas são podadas periodicamente para manejar a entrada de luz em períodos críticos e/ou para aumentar a cobertura morta sobre o solo e incrementar seu teor de matéria orgânica (VIVAN, 2002; GARCEZ; COTRIM, 2007; GARCEZ; MIELITZ NETO, 2008; FAVRETO et al., 2010; BJÖRKLUND et al. 2012; WIVES; MACHADO, 2014; GONCLAVES; MOTTER, 2015). É feito um controle de sombreamento no SAF quando as árvores crescem e ultrapassam as bananeiras, definindo assim uma estrutura vertical típica, que não comprometa a produção de banana (VIVAN, 2002; GONCLAVES; MOTTER, 2015).

Os SAF podem ser estudados e avaliados segundo a abordagem da Silvicultura de Precisão ou Floresta de Precisão, que fazem uso de diversas geotecnologias, a exemplo do posicionamento pelos Sistemas Globais de Navegação por Satélite (GNSS), uso de Sistemas de Informações Geográficas (SIG), banco de dados geográficos, dentre outras inúmeras tecnologias. Essas tecnologias podem ser empregadas, por exemplo, para determinar a variabilidade da produção, dos fatores que a condicionam no tempo e no espaço, bem como de outras características destes sistemas alternativos de produção, para elaborar recomendações de manejo otimizadas e específicas para determinadas zonas dentro da área de cultivo, seja cultivo agrícola ou florestal. Este manejo específico é possível pelo uso de ferramentas especializadas em gestão e visa maximizar a produção e a renda obtida, aplicar de forma eficiente os recursos disponíveis, minimizar os impactos ambientais, dentre outros (BANDELERO et al., 2007; MAEDA et al., 2014; AMARAL et al., 2018).

Nos SAF silvibananeiros do Litoral Norte do Rio Grande do Sul estudos com esta abordagem ainda não foram realizados. Conhecer a variabilidade espacial dos SAF é importante, pois poderá ser utilizada para otimizar o seu manejo. Neste sentido, o geoprocessamento (coleta e armazenamento de dados, análise e geração de informações) e as geotecnologias, associadas a Silvicultura, Manejo Florestal e Exploração Florestal, dão suporte ao que se chama de Floresta de Precisão (BANDELERO et al., 2007; MAEDA et al., 2014; AMARAL et al., 2018).

Os SIG são difundidos por sua capacidade de auxiliar gestores na tomada de decisão e são aliados na formulação de políticas públicas para gestão do território, análise espaciais e ambientais, agricultura de precisão, análise de zoonoses e epidemias, gestão dos recursos hídricos, análise socioeconômica de populações, planejamento urbano e rural, geomarketing, cartografia temática, dentre uma infinidade de possíveis aplicações (LONGLEY et al., 2013; MIRANDA, 2015). A utilização de SIG cresceu consideravelmente nas últimas décadas, possibilitando um melhor gerenciamento de informações, o que proporciona uma melhoria para a tomada de decisões em diversas áreas como planejamentos municipais, estaduais e federais, e também no setor florestal em áreas de preservação ambiental, reservas legais, fragmentação, plantios comerciais, que deverá ser ainda mais massificado neste setor com a adoção da Silvicultura de Precisão ou Foresta de Precisão. Os SIG são importantes geotecnologias, especialmente quando aplicadas à Ciência Florestal, pois é o meio onde o geoprocessamento propriamente dito ocorre, ou seja, onde as análises espaciais, necessárias para a utilização dos dados georreferenciados coletados na floresta ou em outros usos da terra, podem ser realizadas para executar parte de um projeto de Floresta ou Silvicultura de Precisão.

Neste contexto, o gvSIG ${ }^{\circledR}$ é uma importante opção, por ser um SIG livre e de código aberto (open source), sendo distribuído gratuitamente e pode ter suas funções aprimoradas pelos usuários, considerado interessante às universidades e demais instituições públicas, inclusive prefeituras, pois, além dos computadores, não demanda investimento em aquisição, licença de uso e/ou taxas de atualização de versões de software, bem como aquisição de um sistema operacional para computador específico, pois é compatível com Windows, Linux e Mac OS. O gvSIG conta ainda com versões portáteis (executáveis), que podem ser transportadas em dispositivos de memórias externa e utilizados em computadores sem necessitar de instalação (REOLON, 2008; ASOCIACIÓN gvSIG, 2020). Este SIG possui ainda interface amigável, versatilidade e oferece funções de geoprocessamento de camadas de dados geográficos armazenados em estruturas dos tipos vetorial e raster (REOLON, 2008). Segundo o mesmo autor, o gvSIG foi lançado em 2005, e conta com aprimoramento contínuo, tendo sido desenvolvido pelas instituições espanholas Generalitat Valenciana e Iver Tecnologias de la Información S.A., dentre outros colaboradores e cofinanciadores, como a União Europeia.

Para compreender os fatores que interferem na produtividade das culturas e nos atributos da vegetação, a variabilidade espacial passou a ser incorporada no manejo da produção agrícola e florestal. Técnicas de estatística e análise espacial são ferramentas eficientes para auxiliar na tomada de decisão do manejo mais adequado, pois estudam a variabilidade espacial e temporal de atributos do solo, da planta e do clima. Elas propiciam a geração de informação e o compartilhamento/uso da mesma, na forma de mapas 
temáticos, onde é possível interpretá-las e traduzi-las em ações de intervenções nas lavouras ou outras áreas de interesse, aumentando a compreensão da variabilidade da produtividade, ou de outros atributos, e dos fatores que os condicionam. No entanto, este processo depende da análise espacial. Segundo Longley et al. (2013) a análise espacial é, em muitos sentidos, o ponto crucial dos SIG, pois ela inclui todas as transformações, modificações e métodos que podem ser aplicados aos dados geográficos para adicionar valor a eles, apoiar decisões e revelar padrões e anomalias que não são óbvios à primeira vista, principalmente em dados armazenados nas tradicionais tabelas ou quadros de registro de dados.

No escopo desta pesquisa a interpolação espacial é um ponto primordial. Para Miranda (2015) a definição mais simples de interpolação envolve o processo de determinar valores desconhecidos ou não amostrados, de um atributo contínuo no tempo ou no espaço, a partir de seus valores conhecidos ou amostrados. São necessárias duas etapas para a composição do processo de interpolação, sendo a definição de um relacionamento de vizinhança (determinar quais pontos são os vizinhos apropriados) e a definição de qual método calculará os valores até então desconhecidos, o que necessitará de uma função matemática. A escolha desta função é essencial para obter resultados razoáveis, sendo os melhores resultados obtidos quando se utiliza uma função matemática que se comporta de maneira similar ao fenômeno.

Um dos métodos mais conhecidos de interpolação é a Ponderação pelo Inverso da Distância (IDW). Este método utiliza a Lei de Tobler, para realizar estimativas de medidas desconhecidas com uso de médias ponderadas das medidas conhecidas, obtidas por amostragem em pontos próximos. $\mathrm{O}$ valor interpolado para cada ponto de interesse é a média dos valores observados, calculado e ponderado por um peso $w$, que é inversamente proporcional às distâncias que separam os pontos de observação (LONGLEY et al., 2013; MIRANDA, 2015). Este método é considerado exato, por devolver o valor amostral para o atributo $z$ referente às coordenadas dos pontos da malha amostral, sendo aplicado para dados razoavelmente independentes das suas localizações vizinhas, ou seja, sem tendência regional. Seus pontos positivos são a praticidade, a não necessidade de ajustes de semivariogramas experimentais (Geoestatística), e não exige alto número de pontos como na Geoestatística, é facilmente encontrada em SIG, proprietários ou livres. Seus pontos negativos são o não uso de pesos negativos, fazendo com que este interpolador se restrinja a retornar valores não amostrados sempre dentro dos limites dos valores amostrais. Pode também produzir resultados invertidos em áreas de picos, de depressões e fora da área coberta pelos pontos amostrais, e ainda não determina a incerteza associada às estimativas como na Geoestatística. O usuário deve ser cauteloso e ter cuidado ao aceitar os seus resultados, assegurando-se que eles façam sentido em relação ao seu conhecimento do atributo em estudo numa determinada área (LONGLEY et al., 2013; MIRANDA, 2015).

O objetivo deste trabalho foi avaliar a variabilidade espacial em um sistema agroflorestal silvibananeiro no Litoral Norte do Rio Grande do Sul. Os objetivos específicos foram utilizar a interpolação pela ponderação do inverso das distâncias (IDW) para mostrar a variabilidade espacial dos resultados de uma análise fitossociológica e dados do inventário florestal, com uso do gvSIG como sistema de informação geográfica, livre e gratuito, na geração de mapas por análises espaciais.

\section{MATERIAL E MÉTODOS \\ 2.1. Descrição da área de estudo}

O presente estudo foi realizado em um sistema agroflorestal silvibananeiro, com coordenada central de $29^{\circ} 26^{\prime} 18^{\prime \prime}$ S e 49 55’32" O, localizado no município de Três Cachoeiras, no Litoral Norte do estado do Rio Grande do Sul (Figura 1). Na região onde o SAF está inserido a tipologia florestal é conhecida como Floresta Ombrófila Densa Submontana, com características do Bioma Mata Atlântica (IBGE, 2012).

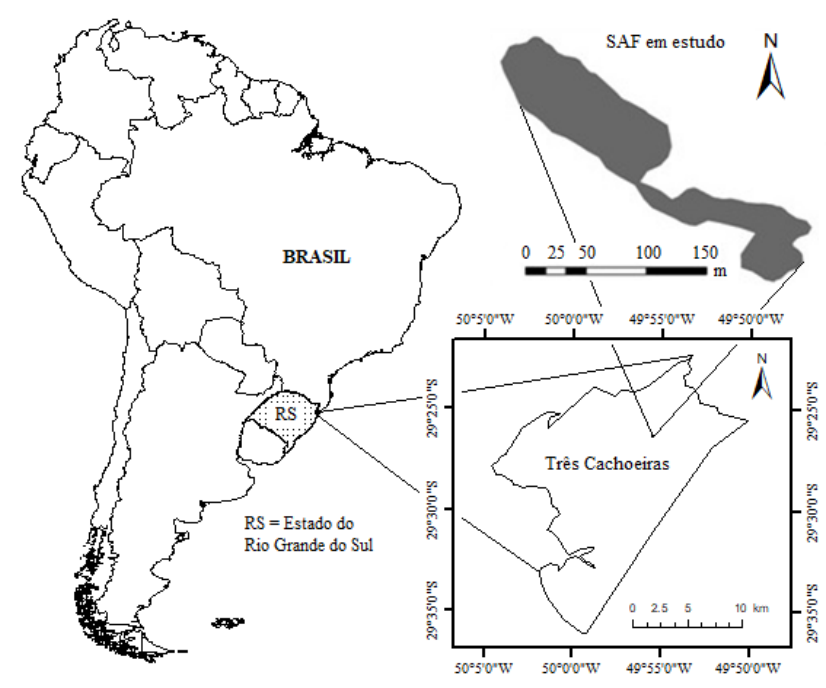

Figura 1. Localização do sistema agroflorestal em estudo em Três Cachoeiras, Litoral Norte do estado do Rio Grande do Sul, Brasil. Fonte: Autores.

Figure 1. Location of agroforestry under study in Três Cachoeiras, North Coast of the State of Rio Grande do Sul, Brazil. Source: Authors.

O clima da região, segundo a classificação por Köppen, é do tipo "Cfa", sendo caracterizado como clima temperado chuvoso e quente, com nenhuma estação seca, com verão quente e mês mais quente com temperatura média maior do que $22^{\circ} \mathrm{C}$ e a do mês mais frio superior a $3^{\circ} \mathrm{C}$, precipitação média anual variando aproximadamente entre 1800 a 1900 mm/ano (WOLLMANN; GALVANI, 2012). As classes de solos predominantes na paisagem da região onde o SAF está localizado são predominantemente Argilosos (podzólico), originados do basalto. O relevo é fortemente ondulado, com boa drenagem e afloramento de rochas em vários pontos (VIVAN, 2002).

\subsection{Coleta de dados georreferenciados, análise fitossociológica e geração de mapas de variabilidade espacial}

Os limites do SAF foram mapeados, com uso de receptor GNSS da marca Garmin ${ }^{\circledR}$, modelo Etrex 66GSX, com erro de precisão posicional de 5 a 15 metros, utilizados para cálculo da área e distribuição das unidades amostrais (ua) do inventário florestal realizado. As coordenadas utilizadas foram do tipo Universal Transversa de Mercator (UTM), referidas ao Sistema Geodésico de Referência mundial WGS 84 (World Geodetic System), Fuso $22 \mathrm{~S}$ e Meridiano Central 51 W (AMARAL et al., 2018). 
Foi utilizado o processo de amostragem aleatória, contendo 33 unidades amostrais (ua) de área variável, definidas pelo método da sexta árvore de Prodan, com os pontos centrais georreferenciados, da mesma forma que os limites do SAF. Para Brito et al. (2007) o método da sexta de Prodan consiste na medição dos diâmetros das seis árvores mais próximas do centro da ua e sua área é calculada pela distância horizontal, que neste caso foi mensurada com trena de fibra de vidro de $20 \mathrm{~m}$, ou raio deste centro até a sexta árvore, no caso a mais distante, adicionado ainda a metade do diâmetro desta sexta árvore, constituindo o raio da $u a$, com o qual se calcula a área das mesmas (Equação 1). Este método foi utilizado pelo fato de permitir dispor as ua no SAF de forma a contemplar todas as regiões em comparação a métodos de amostragem que utilizam unidades amostrais de área maior e/ou área fixa (menor número de ua com maior área amostrada), sendo esta escolha um aspecto positivo do ponto de vista da análise espacial, pois contemplou todas as regiões ou zonas do SAF em estudo.

$$
A=\left(\left(\frac{d}{2}\right)+D\right)^{2} \times \pi
$$

em que: $\mathrm{A}=$ área da unidade amostral $\left(\mathrm{m}^{2}\right) ; \mathrm{d}=$ diâmetro da sexta árvore $(\mathrm{m}) ; \mathrm{D}=$ distância horizontal medida do centro da $u a$ da sexta árvore $(\mathrm{m}) ; \pi=$ número $\operatorname{Pi}(3,141592654)$.

Em cada ua foram identificadas as espécies e contabilizados o número de indivíduos, mensuradas a circunferência à altura do peito em $\mathrm{cm}$ (CAP a partir de 15 $\mathrm{cm}$ ), a altura total em metros, com uso de fita métrica e clinômetro HaglöfHec, respectivamente, sendo contabilizados também os cachos de banana verde, em ponto de corte para comercialização, para descrever a produção de banana no sistema.

A identificação das espécies no SAF em estudo foi feita por Engenheiros Florestais e Técnicos do Centro Ecológico, unidade do Litoral Norte, baseados no sistema Angiosperm Phylogeny Group - APG III (2009), com auxílio dos agricultores. Apesar de se tratar de um sistema agroflorestal silvibananeiro, não foram contados os indivíduos de bananeiras dentro das ua por não serem árvores, somente foram levantados dados relativos à quantidade de cachos de banana verde presentes dentro das $u a$.

Os dados coletados nas 33 ua foram digitados em planilha eletrônica, com os quais foi realizada a análise fitossociológica do SAF em estudo no programa Mata Nativa $2^{\circledR}$ versão 2.10 . A planilha de dados foi importada para o programa, no qual foram realizadas a análise da estrutura horizontal e de diversidade, obtendo-se respectivamente, os parâmetros valor de cobertura (VC) e valor de importância (VI), que definem as espécies mais importantes no SAF em sua estrutura horizontal, e índice de diversidade de ShannonWiener $(H)$, índice de dominância de Simpson $(C)$, índice de Equabilidade de Pielou $(J)$, bem como o número de espécies (S) e a diversidade máxima de espécies $(\ln S)$.

O número de espécies (S) é uma variável importante, pois a partir dela é que se conhece a diversidade da vegetação, determinada pelos índices como o de Shannon-Wiener $\left(H^{\prime}\right)$, equação 2. Este índice estima a diversidade específica, e expressa a heterogeneidade e uniformidade florística da vegetação, geralmente varia de 1,5 a 3,5 nats ind ${ }^{-1}$, raramente apresenta-se acima de 5 nats ind ${ }^{-1}$ (SOUZA; SOARES, 2013).

$$
\mathrm{H}^{\prime}=\frac{\left[(\mathrm{N} \ln (\mathrm{N}))-\left(\sum_{\mathrm{i}=1}^{\mathrm{S}} \mathrm{n}_{\mathrm{i}} \ln \left(\mathrm{n}_{\mathrm{i}}\right)\right)\right]}{\mathrm{N}}
$$

em que: $H^{\prime}=$ índice de Shannon-Wiener, $N=$ número total de indivíduos amostrados; $\ln =$ logaritmo de base neperiana; $\mathrm{S}=$ número total de espécies amostradas; $n i=$ número de indivíduos amostrados da $i$-ésima espécie.

O índice de dominância de Simpson (Equação 3) indica a probabilidade de dois indivíduos, ao acaso, serem da mesma espécie, ou seja, uma vegetação com maior diversidade vai possuir uma menor dominância (SOUZA; SOARES, 2013).

$$
\mathrm{C}=1-\frac{\sum_{\mathrm{i}=1}^{\mathrm{S}} \mathrm{n}_{\mathrm{i}}\left(\mathrm{n}_{\mathrm{i}}-1\right)}{\mathrm{N}(\mathrm{N}-1)}
$$

em que: $C=$ índice de dominância de Simpson; $\mathrm{S}=$ número total de espécies amostradas; $\mathrm{ni}=$ número de indivíduos amostrados da $i$ ésima espécie; $\mathrm{N}$ = número total de indivíduos amostrados.

Já o índice de Equabilidade de Pielou determina a diversidade máxima de espécies. O cálculo (Equação 4) usa entre outros fatores, o índice de Shannon-Wiener (SOUZA; SOARES, 2013).

$$
\mathrm{J}=\frac{\mathrm{H}^{\prime}}{\mathrm{H} \max }
$$

em que: $J=$ índice de equabilidade de Pielou; $H \max =\ln (\mathrm{S}) ; \mathrm{S}=$ número total de espécies amostradas, $H^{\prime}=$ índice de diversidade de Shannon-Wiener.

O programa Mata Nativa ${ }^{\circledR}$ também calcula, no seu módulo de cálculos da estrutura horizontal da vegetação, para cada $u a$, o número de indivíduos $(\mathrm{U})$, a densidade (quantidade de indivíduos de uma determinada espécie na ua) absoluta (DA) e relativa (DR), frequência (número de ua onde uma determinada espécie ocorre) absoluta (FA) e relativa (FR), dominância (quantidade de área basal que uma determinada espécie apresenta em uma ua) absoluta (DoA) e relativa (DoR), valor de cobertura (VC) (Equação 5), que varia de 0 a $200 \%$, e valor de importância (VI) (Equação 6), que varia de 0 a $300 \%$, para todas as espécies, sendo VC e VI expressos de 0 a 100\%, quando divididos, respectivamente, por dois e três, para deixa-los numa mesma escala de medida de grandeza e facilitar seu entendimento (SOUZA; SOARES, 2013).

$$
\mathrm{VC}_{\mathrm{i}}(\%)=\frac{\mathrm{DR}_{\mathrm{i}}+\mathrm{DoR}_{\mathrm{i}}}{2}
$$

em que: $V C_{i}(\%)=$ valor de cobertura da $i$-ésima espécie; $\mathrm{DR}_{\mathrm{i}}=$ densidade relativa da $i$-ésima espécie; DoR $i=$ dominância relativa da $i$-ésima espécie.

$$
\mathrm{VI}_{\mathrm{i}}(\%)=\frac{\mathrm{DR}_{\mathrm{i}}+\mathrm{DoR}_{\mathrm{i}}+\mathrm{FR}_{\mathrm{i}}}{3}
$$

em que: $V I_{i}=$ valor de importância da $i$-ésima espécie; $\mathrm{DR}_{\mathrm{i}}=$ densidade relativa da $i$-ésima espécie; $\operatorname{DoR}_{\mathrm{i}}=$ dominância relativa da $i$-ésima espécie; $\mathrm{FR}_{\mathrm{i}}=$ frequência relativa da $i$-ésima espécie.

Com os dados de contagens de indivíduos das espécies, de maior VI e VC, e dos parâmetros obtidos na análise fitossociológica (estrutura horizontal e diversidade), acrescidas as coordenadas UTM de cada $u a$, foi gerada uma planilha eletrônica para armazenar os dados, salva em arquivo de texto no formato CSV (separado por vírgulas) para ser feita a espacialização (interpolação) dos dados no SIG 
gvSIG $^{\circledR}$ versão 2.3.1.2501 (software livre). Ainda na planilha eletrônica foi realizada a análise de estatística descritiva das variáveis de interesse. Já no programa gvSIG ${ }^{\circledR}$ foi inserida uma nova tabela, com importação da planilha de dados no formato de arquivo CSV. Foi criada uma nova vista e por meio da ferramenta do SEXTANTE, "camada de pontos a partir de uma tabela", foi gerado um arquivo vetorial, de feição pontual, no formato shapefile. Com este arquivo de pontos foram realizadas as interpolações das variáveis, com uso da ferramenta de interpolação pela ponderação do inverso das distâncias (IDW). Este método de interpolação (Equação 7) consiste em estimar medidas desconhecidas utilizando as médias ponderadas, pelo peso $w$ (Equação 8) dos valores de média do conjunto de pontos próximos, sempre enfatizando os pontos mais próximos (LONGLEY et al., 2013).

$$
Z_{(x)}=\frac{\sum_{i} w_{i} z_{i}}{\sum_{i} w_{i}}
$$

em que: $Z_{(\mathrm{x})}=$ valor da variável a ser interpolada em um pixel de localização $\mathrm{x} ; \mathrm{x}=$ localização do pixel onde se quer interpolar um valor de $Z ; Z_{i}=$ valor da variável em análise mensurada em uma $u a$ de localização $\mathrm{x}_{\mathrm{i}} ; i=$ unidades amostrais consideradas.

$$
\mathrm{w}_{\mathrm{i}}=\frac{1}{\mathrm{~d}_{\mathrm{i}}^{2}}
$$

em que: $W_{i}=$ peso de ponderação; $d_{i}=$ distância de $\mathrm{x}_{\mathrm{i}}$ para $\mathrm{x}(\mathrm{xi}=$ localização da unidade amostral onde uma dada variável foi medida e x = localização do pixel onde se quer interpolar um valor de uma dada variável); $i=$ unidades amostrais consideradas.

O resultado do processo de interpolação consiste em superfícies interpoladas, que demonstram a variabilidade espacial das variáveis em análise, armazenadas em arquivos de estrutura matricial (nova imagem com saída do resultado da análise). Com estas superfícies foram elaborados os "mapas" de variabilidade espacial de atributos do SAF silvibananeiro, com as variáveis de interesse.

\section{RESULTADOS}

A área calculada para o SAF em estudo, a partir do levantamento com o receptor GNSS, foi de 1,08 ha. Nesta área foram instaladas e mensuradas 33 unidades amostrais (ua) de área variável, totalizando uma área amostral de 2.549,06 $\mathrm{m}^{2}(0,2549 \mathrm{ha})$, que corresponde a 23,6\% da área total do SAF em estudo. Foram mensurados 198 indivíduos nestas $u a$, que correspondem a 776 indivíduos por hectare, dos quais 14 indivíduos não foram identificados $(7,07 \%$ do total observado), sendo os demais indivíduos distribuídos em 22 espécies, um gênero e 17 famílias botânicas (Tabela 1).

$\mathrm{Na}$ análise da estrutura horizontal do SAF a espécie que mais se destacou em relação à dominância relativa, frequência relativa e densidade relativa foi o palmito juçara (Euterpe edulis Mart.), com valores de 17, 22,2 e 37\%, respectivamente para estes parâmetros $(76,2 \%$ do VI total), que apresentou 75 indivíduos amostrados, seguido pelas espécies cedro (Cedrella fissilis Vell.), canjarana (Cabralea canjerana (Vell.) Mart) e embaúba (Cecropia pachystachya Trécul) (Figura 2), respectivamente com 17, 17 e 12 indivíduos amostrados para cada espécie.

A Figura 2 apresenta o comportamento da dominância, frequência e densidade relativas para as espécies mais abundantes no SAF em estudo, na qual se destaca o palmiteiro ou palmito juçara (E. edulis Mart.).
Tabela 1. Espécies e famílias botânicas dos indivíduos arbóreos inventariados no SAF em estudo no Litoral Norte do Estado do Rio Grande do Sul, Brasil.

Table 1. Species and botanical families of tree individuals inventoried in the SAF under study in the North Coast of the State

\begin{tabular}{|c|c|c|c|}
\hline Nome Científico & Familia & $\mathrm{N}$ & ua $a_{o c \_s p}$ \\
\hline Euterpe edulis Mart. & Arecaceae & 75 & 28 \\
\hline Cedrella fissilis Vell. & Meliaceae & 17 & 15 \\
\hline Cabralea canjerana (Vell.) Mart. & Meliaceae & 17 & 12 \\
\hline Não identificado & N.I. & 14 & 11 \\
\hline Cecropia pachystachya Trécul & Urticaceae & 12 & 7 \\
\hline Zanthoxylum rhoifolium Lam. & Rutaceae & 10 & 8 \\
\hline Daphnopsis fasciculata (Meisn.) Nevling & Thymelaeaceae & 10 & 7 \\
\hline Psidium cattleianum Sabine & Myrtaceae & 7 & 7 \\
\hline Colubrina glandulosa Perkins & Rhamnaceae & 3 & 3 \\
\hline $\begin{array}{l}\text { Albiria niopoides (Spruce ex Benth.) } \\
\text { Burkart }\end{array}$ & Fabaceae & 6 & 3 \\
\hline Citrus sp. & Rutaceae & 5 & 4 \\
\hline Persea americana Mill. & Lauraceae & 4 & 3 \\
\hline Nectandra lanceolata Nees & Lauraceae & 3 & 2 \\
\hline Hovenia dulcis Thunb. & Rhamnaceae & 2 & 2 \\
\hline Jacaranda micrantha Cham. & Bignoniaceae & 2 & 2 \\
\hline Citrus sp. & Rutaceae & 2 & 2 \\
\hline Alchornea triplinervia (Spreng.) M. Arg. & Euphorbiaceae & 1 & 1 \\
\hline Araucaria angustifolia (Bertol.) Kuntze & Araucariaceae & 1 & 1 \\
\hline Erythrina cristagalli $\mathrm{L}$. & Fabaceae & 1 & 1 \\
\hline Hyeronima alchorneoides Allemão & Phyllanthaceae & 1 & 1 \\
\hline Myrsine umbellata Mart. & Primulaceae & 1 & 1 \\
\hline Tibouchina trichopoda (DC.) Baill. & Melastomataceae & 1 & 1 \\
\hline Inga vera Willd. & Fabaceae & 1 & 1 \\
\hline $\begin{array}{l}\text { Cordia trichotoma (Vell.) Arrab. ex } \\
\text { Steud. }\end{array}$ & Boraginaceae & 1 & 1 \\
\hline Eriobotrya japonica (Thunb.) Lindl. & Rosaceae & 1 & 1 \\
\hline Total & & 198 & -- \\
\hline
\end{tabular}
of Rio Grande do Sul, Brazil.

Legenda: $\mathrm{N}=$ número de indivíduos; $\mathrm{ua}_{\mathrm{oc} \_ \text {_esp }}=$ número de unidades amostrais na qual a espécie ocorreu ou frequência; NI = Não identificado.

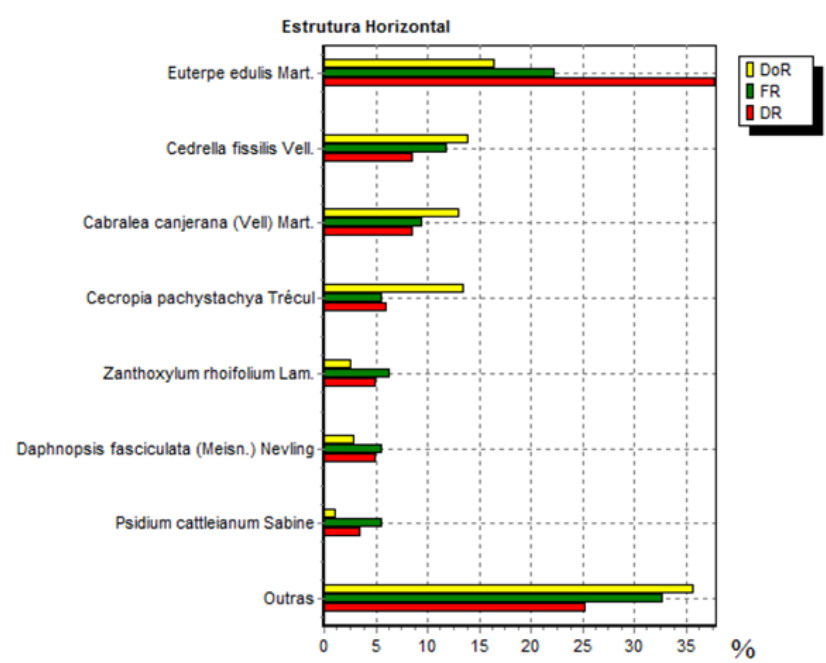

Legenda: DoR $=$ Dominância relativa $; \mathrm{FR}=$ Frequência Relativa; $\mathrm{DR}=$ Densidade relativa; $\%=$ Percentagem.

Figura 2. Parâmetros da estrutura horizontal para espécies arbóreas de um SAF silvibananeiro no Litoral Norte do Rio Grande do Sul, Brasil.

Figure 2. Horizontal structure parameters for tree species of a silvibanan SAF in the North Coast of Rio Grande do Sul, Brazil. 

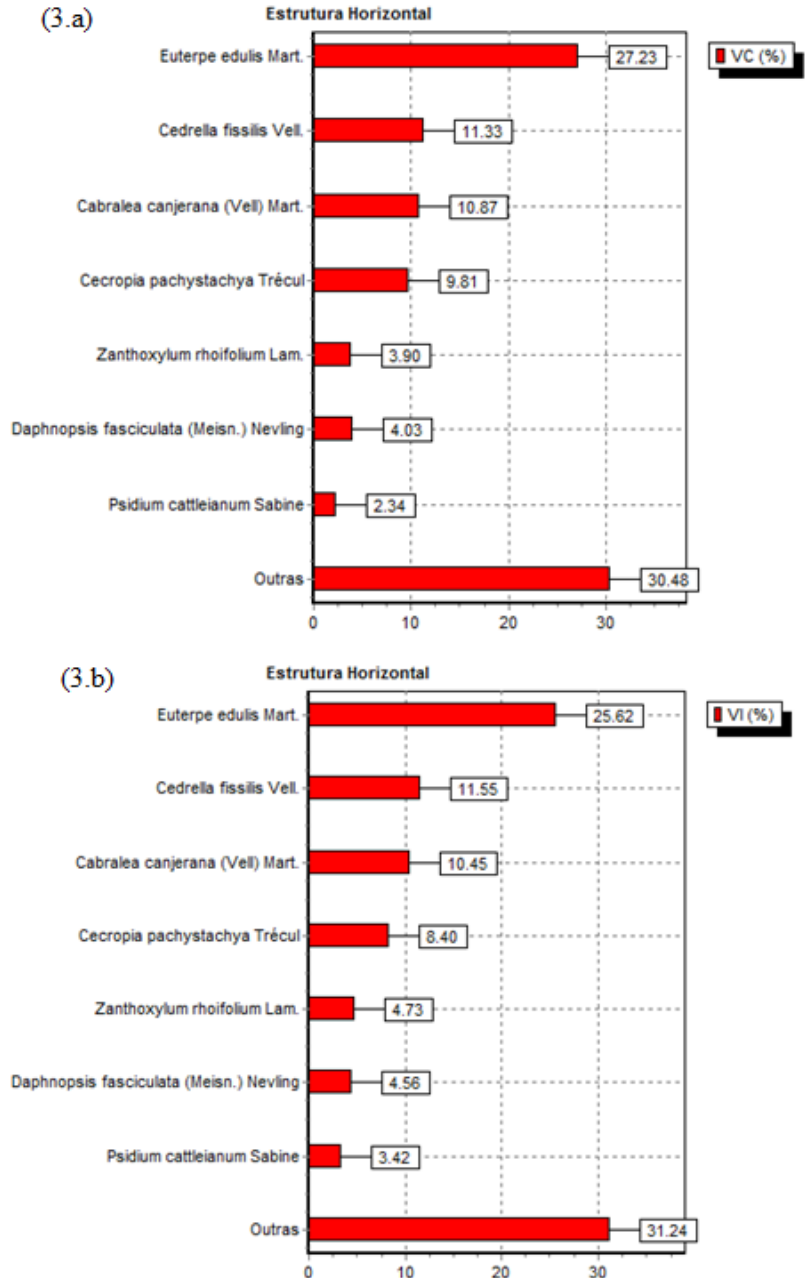

Legenda: VC $(\%)=$ Valor de cobertura em percentagem; VI $(\%)=$ Valor de importância em percentagem.

Figura 3. Valor de cobertura e valor de importância para espécies arbóreas de um SAF silvibananeiro no Litoral Norte do Rio Grande do Sul, Brasil.

Figure 3. Coverage value and importance value for tree species of a silvibanan SAF in the North Coast of Rio Grande do Sul, Brazil.

Quanto aos valores de cobertura (VC) e valores de importância (VI) obtidos para as espécies no SAF em estudo, os mesmos demonstraram que a espécie E. edulis Mart apresentou o maior valor para estes parâmetros, sendo respectivamente de 27,23 e 25,62\%, seguida pelas espécies Cedrella fissilis Vell, Cabralea canjerana (Vell) Mart, Cecropia pachystachya Trécul (Figura 3.a e 3.b). Cabe destacar que se a bananeira fosse uma espécie arbórea, ela seria a de maior VI e VC, por ser a principal cultura comercial do SAF, seguida pelo palmito juçara.

$\mathrm{Na}$ análise de estatística descritiva (Tabela 2) observa-se que a área das unidades amostrais (ua de área variável) apresentou o maior desvio padrão, devido ao fato de haver zonas dentro do SAF com diferentes densidades de árvores, o que afeta a distância entre elas, e por consequência a área das ua. Os parâmetros $\mathrm{S}, \ln (\mathrm{S})$ e os índices $H^{\prime}, C$ e $J$ apresentaram assimetria negativa, tendo mais valores amostrais maiores que a média, sendo esta menor que a mediana e moda, e curtose positiva ou leptocúrtica, e sugere alta homogeneidade nos dados (RIBEIRO JÚRNIOR, 2004). Pode-se observar que a distribuição das ua ao longo do SAF contempla todas as regiões do mesmo (Figura 4.a). Ao analisar a variabilidade espacial do número ou riqueza de espécies (S), pode-se observar uma zona de menor densidade de árvores na parte superior do SAF (Figura 4.b), sendo esta região a mais recentemente manejada na área (área de expansão do limite do SAF) e de maior altitude. A altitude influencia a implantação do SAF, pois acima de 500-600 m a bananeira já começa a ter restrição de cultivo. Nestas elevações também pode ocorrer à mudança de tipologia florestal, de Floresta Ombrófila Densa Submontana para Floresta Ombrófila Densa Montana, e assim poderá haver mudança na composição de espécies. A diversidade máxima de espécies $(\ln (\mathrm{S}))$ apresentou maiores valores na porção central e inferior do SAF (Figura 4.c), sendo esta última a de menor altitude.

Tabela 2. Estatística descritiva dos parâmetros da estrutura horizontal para espécies arbóreas de um SAF silvibananeiro no Litoral Norte do Rio Grande do Sul, Brasil.

Table 2. Descriptive statistics of the horizontal structure parameters for tree species of a SAF silvibanan on the north coast of Rio Grande do Sul, Brazil.

\begin{tabular}{cccccccc}
\hline Par. & M & Md & Mo & S' & Cur & As & CV\% \\
\hline A & 77,24 & 65,55 & Amd & 52,6 & 9,7 & 2,6 & 68,14 \\
S & 3,79 & 4 & 4 & 1,1 & 0,3 & $-0,1$ & 29,34 \\
$\ln (\mathrm{S})$ & 1,28 & 1,39 & 1,39 & 0,3 & 4,4 & $-1,6$ & 27,5 \\
H' & 1,17 & 1,24 & 1,24 & 0,4 & 1,6 & $-0,9$ & 32,37 \\
C & 0,75 & 0,80 & 0,80 & 0,2 & 5,3 & $-1,9$ & 26,64 \\
J & 0,87 & 0,92 & 0,89 & 0,2 & 19,4 & $-4,0$ & 20,21 \\
CB & 1,42 & 2 & 0 & 1,3 & $-1,0$ & 0,3 & 89,56 \\
E1 & 2,27 & 2 & 3 & 1,5 & $-0,4$ & 0,3 & 68,05 \\
E2 & 0,52 & 0 & 0 & 0,6 & $-0,3$ & 0,8 & 120,1 \\
E3 & 0,52 & 0 & 0 & 0,8 & 1,8 & 1,5 & 154,4 \\
E4 & 0,36 & 0 & 0 & 0,9 & 9,9 & 3,1 & 246,1 \\
\hline
\end{tabular}

Legenda: Par. = Parâmetros fitossociológicos; $\mathrm{M}=$ Média; $\mathrm{Md}=$ Mediana; Mo = Moda; $\mathrm{S}^{\prime}=$ Desvio padrão; Cur $=$ Curtose; As = Assimetria; $\mathrm{CV} \%=$ Coeficiente de variação; $\mathrm{A}=$ Área em metros quadrados; $\mathrm{S}=$ Número de espécies ou riqueza de espécies; $\ln (\mathrm{S})=$ Diversidade máxima de espécies; $H^{\prime}$ = Índice de Shannon-Wiener; $C=$ Índice de dominância de Simpson; $J=$ Índice de equabilidade de Pielou; $\mathrm{CB}=$ cachos de banana verde em ponto de corte; E1 = Euterpe edulis Mart.; E2 = Cedrella fissilis Vell.; E3 = Cabralea canjerana (Vell) Mart; E4 = Cecropia pachystachya Trécul.

$O$ índice de Shannon-Wiener $(H)$, que representa a diversidade florística da população, variou de 0 a 1,79 nats indivíduo- $^{-1}$ (Figura 4.d), que significa baixa diversidade de espécies arbóreas individualmente por ua. No entanto, o H' geral, ou seja, a diversidade de espécies em relação a todos os indivíduos arbóreos amostrados foi de 2,16 nats indivíduo-1, aproximando-se de uma média diversidade. $O$ índice de dominância de Simpson (C) gerou resultados entre 0 a 1 , onde quanto mais próximo ao valor de um, maior a diversidade dentro da unidade, ou seja, menor a dominância entre as espécies (Figura 4.e). Para o índice de equabilidade de Pielou (J), os resultados também variam de 0 a 1 , onde valores mais próximos a um representaram uma grande diversidade, ou seja, o mapa demonstrou haver uma grande variabilidade de indivíduos arbóreos. Em outras palavras, este índice demonstrou a heterogeneidade dentro dos seis indivíduos medidos em cada ua pelo método da sexta de Prodan (Figura 4.f).

Ao observar a variabilidade espacial do número de indivíduos de Euterpe edullis Mart. (Figura 4.h) nota-se que na região superior da área, onde houve maior concentração da espécie, foi consequentemente onde obteve-se os menores índices de diversidade, logo também ocorreu maior dominância de uma espécie (Figura 4.e), sendo também a região com os menores números de espécies (Figura 4.b), menor diversidade máxima (Figura 4.c), menor diversidade (Figura 4.d) e menor equabilidade (Figura 4.f). 

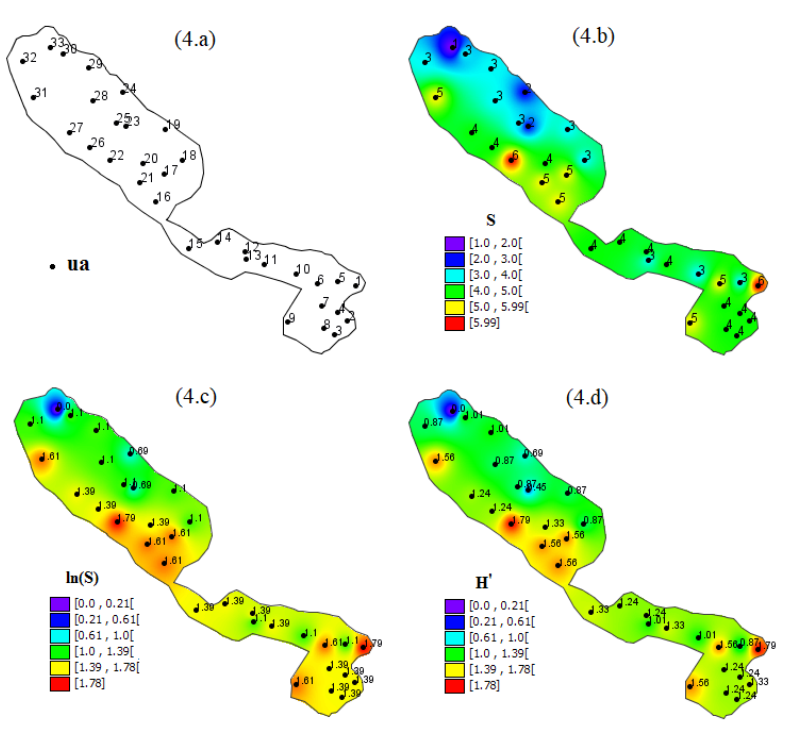

(4.e)
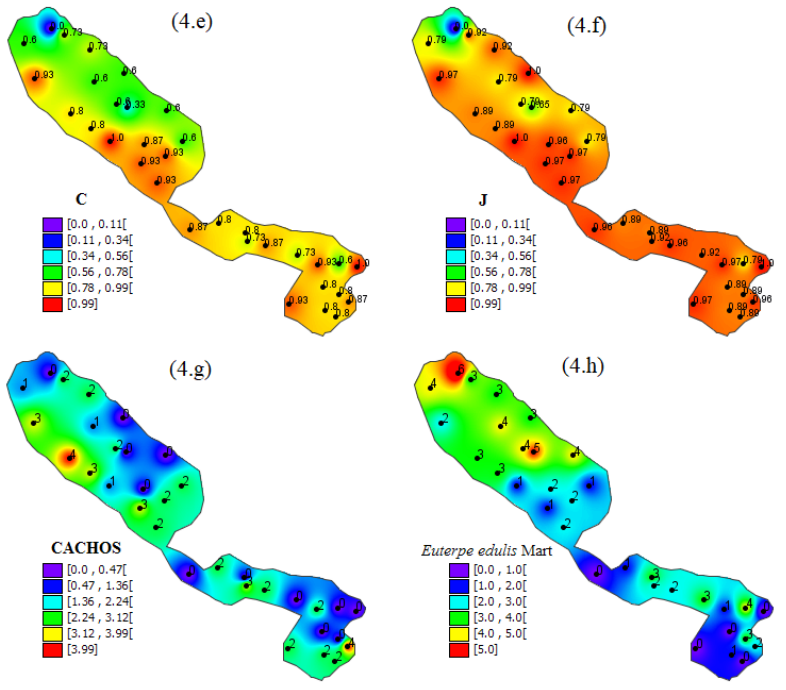

(4.i)
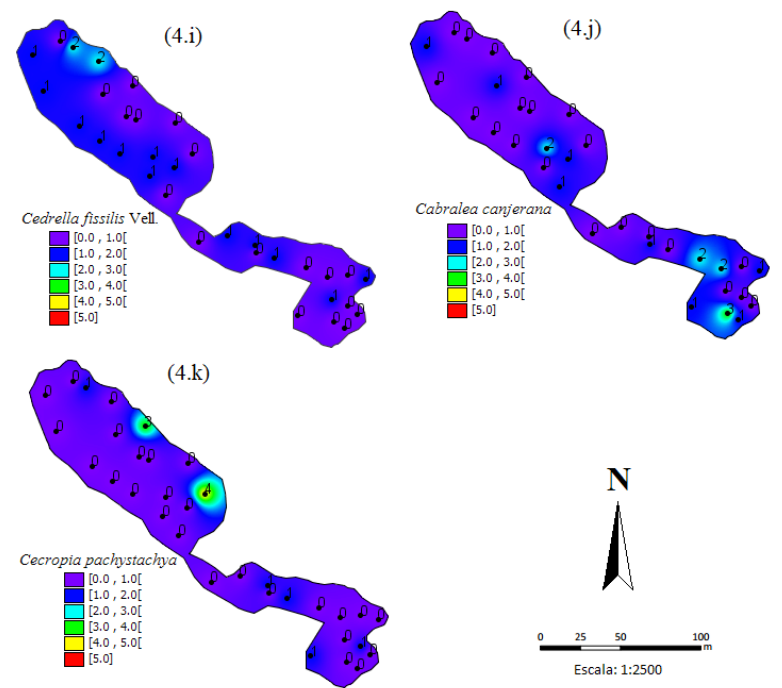

Legenda: $u a=$ Unidade amostral; $\mathrm{S}=$ Número de espécies; $\operatorname{Ln}(\mathrm{S})=$ Diversidade máxima; $H^{\prime}$ = Índice de diversidade de Shannon-Wiener; $\mathrm{C}=$ Índice de dominância de Simpson; $J$ = Índice de equabilidade de Pielou; Cachos = Cachos de banana verde em ponto de corte.

Figura 4. Mapas de variabilidade espacial de um SAF silvibananeiro no Litoral Norte do Rio Grande do Sul, Brasil.

Figure 4. Spatial variability maps of a silvibanan SAF in the North Coast of Rio Grande do Sul, Brazil.

Os mapas de variabilidade espacial das espécies foram gerados apenas para as quatro espécies que tiveram o maior
VI, sendo Euterpe edulis Mart. (Figura 5.h), Cedrella fissilis Vell (Figura 4.i), Cabralea canjerana (Vell) Mart (Figura 4.j) e Cecropia pachystachya Trécul. (Figura 4.k), onde o palmito juçara Euterpe edulis Mart, chamou atenção por estar em maior concentração na região de maior altitude do SAF (porção superior do limite do SAF no mapa).

\section{DISCUSSÃO}

O cultivo de bananeiras em consórcio com juçara, ou ripeira (como é chamado o palmiteiro pelos agricultores da região), e floresta secundária é uma prática agroflorestal que não é exclusiva do Litoral Norte do Rio Grande do Sul. Danelli et al. (2016) relataram em seu trabalho o manejo de bananeiras e juçara em florestas secundárias no município de Camboriú, litoral de Santa Catarina, e também na região nordeste do Estado de São Paulo, onde se faz cultivo de bananeiras em consórcio com juçara e floresta secundária em unidades de conservação, mais especificamente, do Parque Estadual da Serra do Mar, ou no entorno das mesmas.

A composição de espécies e a expressividade do palmito juçara (E. edulis) observada nesta pesquisa (Tabela 1) está coerente como à descrita pela literatura para SAF silvibananeiro no Litoral Norte do Rio Grande do Sul em diversos trabalhos (VIVAN, 2002; GARCEZ; COTRIM, 2007; FAVRETO et al., 2010; GONÇALVES; MOTTER, 2015 e AMARAL et al. (2018). Gonçalves et al. (2013) avaliaram SAF manejados por 16 produtores rurais, distribuídos em quatro municípios no Litoral Norte do Rio Grande do Sul (Três Cachoeiras, Mampituba, Dom Pedro de Alcântara e Morrinhos do Sul), utilizando 44 ua de área fixa de $700 \mathrm{~m}^{2}$, nas quais foram amostrados 1339 indivíduos arbóreos, distribuídos em 30 famílias botânicas e 71 espécies, das quais as mais importantes foram Euterpe edulis Mart., Cecropia pachystachya Trécul, Cabralea canjerana (Vell) Mart., com valor de importância de 75,85 (25,28\%), 35,79 (11,93\%) e $12,35(4,65 \%)$ respectivamente, totalizando $41,31 \%$ do VI observado. Estes dados corroboram a estrutura horizontal do SAF observada no presente trabalho (Figura 2; Figura 3.a e 3.b). Na época da realização do levantamento descritos pelos autores supracitados, a grande maioria dos indivíduos de embaúba (C. pachystachya) apresentavam grande porte, e estavam sendo suprimidos do sistema para proporcionar maior entrada de luz (manutenção do índice de 40 - 50\% de sombreamento para não diminuir a produção de banana), possivelmente a causa para esta espécie ter apresentado a quarta posição para VI no atual estudo (Figura 3.b), por ser posterior aos descritos acima. A questão do manejo do sombreamento é necessária para propiciar a produção de banana e foi descrita por Vivan (2002) e Gonçalves; Motter (2015).

Segundo Vivan (2002) no Litoral Norte do Rio Grande do Sul os bananais em SAF priorizam o palmiteiro quanto ao uso de palmeiras, e sua importância no dossel dominante varia entre 17 a $80 \%$. Esta espécie possui alta densidade de plantas no SAF, pois foi introduzida a partir da semeadura de sementes pré-germinadas, a lanço ou plantadas em sulcos, oriundas da despolpação mecânica dos frutos com água morna, o que quebra a dormência e estimula a germinação. No SAF, o palmiteiro se beneficia da sombra, da umidade e da matéria orgânica no solo, oriunda da biomassa das bananeiras mortas, e serve ainda de alimento para avifauna, que auxiliará na dispersão de outras espécies da Mata Atlântica (TROIAN et al., 2014). Favreto et al. (2010) 


\section{Amaral et al.}

demonstraram em sua pesquisa que o palmiteiro cresce e desenvolve-se melhor no bananal do que na floresta secundária, ao comparar dados obtidos em parcelas de plantios desta espécie nestes dois usos da terra em Maquiné, Litoral Norte do Rio Grande do Sul, cinco anos após o plantio. Os autores observaram que no bananal o palmiteiro apresentou maior diâmetro da base, maior altura, melhor qualidade das folhas e menor mortalidade acumulada de indivíduos jovens. Esta é uma espécie com muito potencial comercial, pois seus produtos, tais como polpa de frutos (o chamado "açaí de juçara" ou "açaí da Mata Atlântica") e palmito, tem um variado e diversificado mercado de consumo como programas de aquisição de alimentos do governo federal, suco, sorvetes, doces, geleias, feira-livre, dentre outras opções (TROIAN et al., 2014; GONÇALVES; MOTTER, 2015).

As demais espécies cumprem as funções de produção de madeira, frutas e fornecimento de sombra e material vegetal para cobertura do solo, mediante o uso de podas das copas. O cedro (Cedrella fissilis Vell) e canjarana (Cabralea canjerana (Vell) Mart) são espécies da família Meliaceae e cultivadas no SAF com fins madeireiros, assim como o loro pardo (Cordia trichotoma (Vell.) Arrab. ex Steud., família Boraginaceae). Já a embaúba (Cecropia pachystachya Trécul.) é utilizada na função de sombra e manejo de biomassa e ciclagem de nutrientes, mediante podas ou retirada do sistema com distribuição de sua biomassa no SAF, em cobertura ao solo (VIVAN, 2002; GONCLAVES; MOTTER, 2015).

Quanto aos índices de diversidade (valor geral 2,16 nats indivíduo ${ }^{-1}$ ), dominância e equabilidade refletem uma diversidade média. Os mesmos também foram coerentes com os obtidos por Gonçalves et al. (2013), que observaram para SAF silvibananeiro na região de Torres-RS um $H^{\prime}$ geral de 2,74 nats ind ${ }^{-1}$, sendo considerado médio, tendo um índice de dominância $(C)$ de 0,81 , o que significa que havia dominância de algumas espécies na ocorrência dos indivíduos amostrados, neste caso as comerciais, Euterpe edullis Mart. e Musa sp. Já o índice de equabilidade geral ( $($ ) foi de 0,61 e indicou que havia dominância de poucas espécies, assim como o anterior. Ressalta-se que a diversidade de espécies arbóreas, juntamente com a fixação de gases de efeito estufa (fixação de carbono), tem sido considerada como serviços ambientais provido pelos SAF na região, especificamente pelos SAF silvibananeiros no Litoral Norte do Rio Grande do Sul em relação aos bananais convencionais lá cultivados (BJÖRKLUND et al., 2012; GONÇALVES et al., 2013).

A estatística descritiva foi realizada para dar suporte ao mapeamento da variabilidade espacial dos atributos da vegetação no SAF. As variáveis obtidas nas ua, número de espécies (S), diversidade máxima $(\ln (S))$ e os índices de diversidade, dominância e equabilidade apresentaram valores próximos para média, mediana e moda, sugerindo haver normalidade nos dados. Segundo Andriotti (2003), os coeficientes de variação $(\mathrm{CV} \%)$ inferiores a $40 \%$ indicam homogeneidade nas $u a$, já índices superiores a 100\% indicam que a amostra tem grande heterogeneidade. $\mathrm{O} \mathrm{CV} \%$ dos índices de diversidades de Shannon-Wiener, dominância de Simpson, equabilidade de Pielou ( $H^{\prime}, C$ e $\left.J\right)$, assim como o do número de espécies $(\mathrm{S})$ e da diversidade máxima de espécies $(\ln (\mathrm{S}))$, demonstraram uma baixa variabilidade.

Conforme os mapas de variabilidade espacial gerados, as unidades amostrais foram bem distribuídas na área do SAF (Figura 5.a), e o número de espécies (Figura 5.b), a diversidade máxima (Figura 5.c), índice de diversidade
(Figura 5.d), índice de dominância (Figura 5.e), equabilidade (Figura 5.f), cachos de banana (Figura 5.g) e número de indivíduos de palmiteiro (Figura 5.h) apresentaram mapas com variação espacial evidente ao longo do SAF. Já cedro, canjarana e embaúba apresentaram ocorrência pontual no SAF em análise (Figura 4.i; 4.j e 4.k).

Para a variável cachos de banana (Figura 4.g), observouse que eles ficaram relativamente bem distribuídos no mapa, com um número total de 47 cachos observados no SAF, média de 1,42 cachos por $u a$, o que corresponde a 184 cachos ha $^{-1}$. As bananeiras são plantadas no SAF com um espaçamento de $2,5 \times 2,5 \mathrm{~m}$, totalizando uma densidade de 1600 plantas por hectare (VIVAN, 2002; GONÇALVES e MOTTER, 2015), mas com o desenvolvimento das plantas vão surgindo vários pseudocaules que facilitam a ocupação da área por esta frutífera, sendo a espécie de maior densidade no SAF, devido ser a principal cultura comercial do mesmo.

As descrições da variabilidade espacial das variáveis analisadas no SAF podem ser fontes de informação para a definição de zonas de manejo diferenciadas. As geotecnologias e os dados geográficos podem e devem ser somados ao conhecimento dos agricultores agroflorestais desta região, que segundo Vivan (2002), já fazem distinção de zonas no bananal, a ser transformado em SAF, quanto a nutrientes, entrada de luz e umidade, regrando também a composição de espécies arbóreas para sombrear as demais plantas dentro do limite de 50\% de sombreamento, havendo ainda o ajuste de um manejo mais adequado a cada zona, porém isso ainda não foi mapeado e estudado espacialmente.

Uma das primeiras geotecnologias a ser utilizada em SAF foram os receptores GNSS de navegação, que permitem determinar a localização, calcular o perímetro e a área dos SAFs, geralmente em SIG (STEEMBOCK et al., 2013), seguidos da geração de banco de dados para SIG e localização de indivíduos arbóreos em SAF (AMARAL et al., 2018). Ao longo do tempo, isso permitiu ao Centro Ecológico disponibilizar um webSIG (talvez um dos primeiros no Brasil, em funcionamento desde 2014), voltado para serviços ambientais gerados por SAFs, no quais foram mensurados diversidade de espécies arbóreas, e estimados biomassa acima do solo e carbono estocado nos SAF silvibananeiros do Litoral Norte do Rio Grande do Sul (http://www.centroecologico.org.br/safs_sig.aspx).

Recentemente as pesquisas que abordam Floresta de Precisão e os estudos de variabilidade espacial nos SAFs começaram a surgir, e de maneira análoga a forma como aconteceu na agricultura de precisão, ao basearem em princípios comuns (FARDUSI et al., 2017), a maior parte das pesquisas abordam os atributos físicos e químicos do solo, a exemplo do trabalho de Soares et al., (2018), que analisaram a variabilidade de atributos do solo em SAF na Amazônia, e atualmente a atenção se volta para diversos aspectos da vegetação ou localização dos SAF (BOLF, 2019), assim como a presente pesquisa.

As geotecnologias, o geoprocessamento e a abordagem de Floresta de Precisão podem auxiliar neste processo, havendo relatos na literatura destas possibilidades associadas à silvicultura. Brandelero et al. (2007), Maeda et al. (2014) e Corona et al. (2018) enfatizam a importância do uso de sistemas GNSS e sistemas de informações geográficas, dentre outras ferramentas, para as atividades florestais com abordagem de Floresta de Precisão.

Figueiredo et al. (2007) associaram ao Manejo Florestal de Precisão o uso de mapas de localização das árvores em 
geral, dos indivíduos selecionados, da infraestrutura e dos acessos da floresta, gerados em SIG a partir de mapeamento de inventários florestais 100\%. Estes mapas apoiam as intervenções na floresta, visando proteger e preservar árvores individuais, e coletivamente, conservar e elevar a biodiversidade. Souza e Soares (2013) descrevem e apresentam um mapa de inventário $100 \%$ na Unidade de Produção Anual 1 e Área de Manejo Florestal da Vila Céu do Mapiá, Flona do Purus, AM, baseados no trabalho de Brandão (2005), no qual todas as árvores de interesse foram georreferenciadas com receptor GNSS de navegação. Os autores também apresentaram um exemplo de consulta e seleção de dados não espaciais (atributos das árvores mapeadas e inventariadas), lidos na tabela de atributos de um arquivo vetorial shapefile, de feição pontual para representar as árvores, no sig ArcGis. Esse tipo de consulta e seleção de dados em SIG, também foi demonstrada por Amaral et al. (2018), aplicado a SAF silvibananeiro no Litoral Norte do Rio Grande do Sul.

Desta forma, os resultados obtidos nesta pesquisa, estão alinhados ao pensamento de Kovácsová; Antalová (2010), que consideram Floresta de Precisão, aquelas que são produzidas com uso de ferramentas e técnicas modernas, para obter o maior número possível de informações reais, com melhorias no processo de tomada de decisão e garantir que as metas de manejo florestal sejam cumpridas. A tendência é que trabalhos como os citados anteriormente, bem como o descrito na presente pesquisa, sejam cada vez mais utilizados nos SAF e em outros sistemas alternativos de uso da terra para dar suporte à tomada de decisão quanto ao manejo e intervenção, monitoramento e inventários para serviços ambientais, dentre outras aplicações.

\section{CONCLUSÕES}

Os atributos da vegetação do sistema agroflorestal silvibananeiro que apresentaram variabilidade espacial evidente, se comportando de maneira continua no SAF, foram número de espécies ( $\mathrm{S}$ ), diversidade máxima de espécies $(\ln (\mathrm{S}))$, índice de diversidade de Shanonn-Wiener $(H)$, índice de dominância de Simpson $(C)$, índice de equabilidade de Pielou $(J)$, número de cachos de banana verde em ponto de corte e número de indivíduos de palmiteiro ou juçara (ripeira). Os mapas de variabilidade espacial, gerados por ponderação pelo inverso da distância (IDW), espacializaram de maneira apropriada a vegetação do SAF em estudo.

Euterpe edulis Mart. (palmiteiro ou ripeira) apresentou maior relevância dentre as espécies arbóreas levantadas no SAF, e seu mapa foi um dos de maior destaque nesta pesquisa. O palmiteiro e as bananeiras (Musa sp.), são as principais espécies comerciais deste sistema. Também se destacaram as espécies cedro, canjarana e embaúba, mas estas não ocorrem de forma contínua no SAF.

As variáveis relativas à diversidade de espécies em SAF podem ser mapeadas quanto à variabilidade espacial, e tem potencial para serem consideradas como métricas em monitoramento de serviços ambientais. Estas foram mapeadas com relativa facilidade, com base na metodologia e resultados apresentados nesta pesquisa, bem como as variáveis que descrevem a produção do $\mathrm{SAF}$, número de cachos de banana verde e número de indivíduos de Euterpe edullis Mart.. É recomendado realizar também a contagem e mapeamento dos cachos de frutos do palmiteiro, para melhor caracterizar a produtividade dos SAF.
A metodologia proposta mostrou-se adequada para verificar a variabilidade espacial da vegetação que caracteriza o SAF silvibananeiro no Litoral Norte do Rio Grande do Sul. O método da sexta árvore de Prodan mostrou-se bastante eficaz na amostragem, sendo seu uso idealizado para suprir demandas de dados da análise espacial, pois distribuiu as $u a$ em toda área de estudo, recobrindo aproximadamente 1/4 da área total. O uso do gvSIG mostrou-se relativamente fácil e atendeu todas as demandas da pesquisa. No entanto, algumas configurações de tabelas e importações de dados poderiam ser mais facilitadas, quanto à formatação e extensão de arquivos. O uso do interpolador IDW também se mostrou eficiente.

\section{AGRADECIMENTOS}

Ao Centro Ecológico Litoral (Dom Pedro de Alcântara, RS) e seus colaboradores pelo apoio a este estudo, aos agricultores Tobias Fernandes e Paulo Fernandes, por permitirem a realização desta pesquisa em seu Sistema Agroflorestal.

\section{REFERÊNCIAS}

AMARAL, L. P.; FERREIRA, R. A.; SEBEM, E.; RODRIGUES, G.; MOTTER, C. Geração de um banco de dados geográficos em sistema agroflorestal silvibananeiro no Litoral Norte do Rio Grande do Sul, Brasil. In AMARAL, L. P. (Org). Livro Digital: Trabalhos completos do IV Congresso SulAmericano de Agricultura de Precisão e Máquinas Precisas. 1ed. Santa Maria: CESPOL, 2018, p. 52-61.

ANDRIOTTI, J. L. S. Fundamentos de estatística e geoestatística. São Leopoldo: Unisinos, 2003, 165p.

ASOCIACIÓN GVSIG. Conoce gvSIG Desktop, el Sistema de Información Geográfica libre. Disponível em: $\quad<$ http://www.gvsig.com/es/productos/gvsigdesktop>. Acesso em: 19 jun. 2020.

BJÖRKLUND, J.; ARAYA, H.; EDWARDS, S.; GONÇALVES, A.; HÖÖK, K.; LUNDBERG, J.; MEDINA, C. Ecosystem-based agriculture combining production and conservation - A viable way to feed the world in the long term? Journal of Sustainable Agriculture, v. 36, n. 7, p. 824-855, 2012. http://dx.doi.org/10.1080/10440046.2012.705813

BOLF, E. L. Applications of satellite images and Field databases to analyze agroforestry systems in Brazil. International Journal of Advanced Engineering Research and Science, v. 6, n. 12, p. 458-463, 2019. https://dx.doi.org/10.22161/ijaers.612.53

BRANDELERO, C.; ANTUNES, M. U. F.; GIOTTO, E. Silvicultura de Precisão: nova tecnologia para o desenvolvimento florestal. Revista Ambiência, Guarapuava, v. 3, n. 2, p. 269-281, 2007.

CORONA, P.; CHIANUCCI, F.; GROTTI, M.; QUATRINI, V.; PULETTI, N.; MATTIOLI, W. Precision Forestry. Agriregionieuropa, v. 14, n. 53, $2018 . \quad$ Disponível em: $<$ https://agriregionieuropa.univpm.it/it/content/article /31/53/precision-forestry>. Acesso em: 22 jun. 2020.

DANELLI, M. F. D.; FISCH, S. T. V.; VIEIRA, S. A. Análise da estrutura e biomassa florestal de áreas de colheita de frutos de juçara (Euterpe edulis Mart.) no Litoraral Norte e Serra do Mar - SP. Ciência Florestal, Santa Maria, v. 26, n. 3 , p.773-786, $2016 . \quad$ DOI: https://doi.org/10.5902/1980509824206 
FAVRETO, R.; MELLO R. S. P.; BAPTISTA, L. R. M. Growth of Euterpe edulis Mart. (Arecaceae) under Forest and agroforestry in southern Brazil. Agroforest Systems, Amsterdan, v. 80, p. 303-313, 2010. DOI: https://doi.org/10.5902/1980509824206

FARDUSI, M. J.; CHIANUCCI, F.; BARBATI, A. Concept to practices of geospatial information tools to assist forest management and planning under precision forest framework: a review. Annals of Silvicultural Research, v. 41, n. 1, p. 3-14, 2017. DOI: http://dx.doi.org/10.12899/asr-1354

GARCEZ, D.; COTRIM, D. A bananicultura na microrregião do Litoral Norte do Rio Grande do Sul. Revista Brasileira de Agroecologia, v. 2, n. 2, p. 78-81, 2007.

GARCEZ, D.; MIELITZ, C. G. A. Sistema de produção de base ecológica: re-localização e reativação do espaço rural no Litoral Norte do Rio Grande do Sul. Agrária, São Paulo, n. 8, p. 23-48, 2008. DOI: https://doi.org/10.11606/issn.1808-1150.v0i8p23-48

GONÇALVES, A. L. R.; AMARAL, L. P.; PEREIRA Jr., C.; RAMOS, J. B., ATANASIO, W. M.; MOTTER, C.; BELLÉ, N.; FERREIRA, R. A. Serviços ambientais na região de Torres, RS, Brasil. In: CONGRESSO BRASILEIRO DE SISTEMAS AGROFLORESTAIS, 9., 2013, Ilhéus/BA. Anais... Ilhéus/BA: Sociedade Brasileira de Sistemas Agroflorestais, 2013. p. 1-4.

GONÇALVES, A. L. R.; MOTTER, C. Produção ecológica de banana. Torres: Centro Ecológico, 2015, 76 p. Disponível em: <http://www.centroecologico.org.br/cartilhas/Cartilha _Banana_CE.pdf $>$. Acesso: 22 jun. 2020.

IBGE_Instituto Brasileiro de Geografia e Estatística, IBGE. Manual Técnico da Vegetação Brasileira. 2 ed. Rio de Janeiro: IBGE, 2012.274 p.

KOVÁCSOVÁ, P.; ANTALOVÁ, M. Precision Forestry Definition and Technologies. Šumarski list, v. 134, n. 11-12, p. 603-611, 2010.

LONGLEY, P. A.; GOODCHIL, M. F.; MAGUIRE, D. J.; RHIND, D. W. Sistemas e Ciência da Informação Geográfica. 3 ed. Porto Alegre: Bookman, 2013. 540 p.

MAIA, S. M. F.; XAVIER, F. A.S.; OLIVEIRA, T. S.; MENDONÇA, E. S.; ARAÚJO FILHO, J. S. Impactos dos sistemas agroflorestais e convencional sobre a qualidade do solo no Semi-Árido Cearense. Revista Árvore, Viçosa, v. 30, n. 5, p. 837-848, 2006. DOI: https://doi.org/10.1590/S0100-67622006000500018

MAEDA, S.; AHRENS, S.; CHIARELLO, S. R.; OLIEVEIRA, E. B.; STOLLE, L.; FOWLER, J. A. P.; BOGNOLA, I. A. Silvicultura de Precisão. In: BERNARDI, A. C. C.; NAIME, J. M.; RESENDE, A. V.; BASSOI, L. H.; INAMASU, R. Y. (Eds.). Agricultura de Precisão - Resultados de um novo olhar. Brasília: Embrapa Instrumentação, 2014, p. 467477.
MIRANDA, J. I. Fundamentos de sistemas de informações geográficas. 4. ed. Brasília: Embrapa, 2015, 399 p.

NUNES, P.; VIVAN, J. L. Florestas, sistemas agroflorestais e seus serviços ambientais e econômicos em Juruena-MT. Cuiabá: Projeto Poço de Carbono Juruena/ADERJUR, 2011, 40p.

REOLON, C. A. Geotecnologias à Cartografia Temática: gvSIG. Marechal Cândidon Rondon: Associação dos Geógrafos Brasileiros, 2008, 54p.

RIBEIRO JÚNIOR, J. I. Análises estatísticas no Excel: Guia Prático. Viçosa: UFV, 2004, 251p.

SILVA, I. C. Sistemas Agroflorestais: Conceitos e métodos. Itabuna: SBSAF, 2013, 308p.

SOARES, M. D. R.; CAMPOS, M. C. C.; OLIEVIRA, I. A.; CUNHA, J. M.; SOUZA, Z. M.; AQUINO, R. E.; SILVA, D. P.; SILVA, J. F. Variabilidade espacial dos atributos do solo sob agrofloresta na região de Humaitã, AM. Gaia Scientia, João Pessoa, v. 12, n. 1, p. 33-41, 2018. DOI: https://doi.org/10.22478/ufpb.1981$1268.2018 \mathrm{v} 12 \mathrm{n} 1.34353$

SOUZA, A. L.; SOARES, C. P. B. Florestas nativas: estrutura, dinâmica e manejo. Viçosa: Editora UFV, 2013, 322 p.

STEENBOCK, W.; SILVA, R. O.; FROUFE, L. C. M.; SEOANE, C. E. Agroflorestas e sistemas agroflorestais no espaço e no tempo. In: STEENBOCK, W.; SILVA, L. C.; SILVA, R. O.; RODRIGUES, A. S.; PEREZCASSARINO, J.; FONINI, R (Org.). Agrofloresta, ecologia e sociedade. Curitiba: Editora Kairós, 2013, p. 39-60.

TROIAN, L. C.; CORBELLINI; L. C.; PRINTES, R. C.; STOFFEL, A. S. Cartilha da Juçara (Euterpe edulis): Informações sobre Boas Práticas e Manejo. 2014, 35p. DOI: 10.13140/RG.2.2.31931.62245

VIVAN, J. L. Bananicultura em sistemas agroflorestais no Litoral Norte do RS. Agroecologia e Desenvolvimento Rural Sustentável, Porto Alegre, v. 3, n. 2, p. 17-26, 2002.

WIVES, D. G.; MACHADO, J. A. D. Fatores influentes e a tomada de decisão nos sistemas de produção de banana no Litoral Norte do Rio Grande do Sul. Revista Brasileira de Gestão e de Desenvolvimento Rural, Taubaté, v. 10, n. 4, p. 225-247, 2014.

WOLLMANN, C. A.; GALVANI, E. Caracterização climática regional do Rio Grande do Sul: dos estudos estáticos ao entendimento da gênese. Revista Brasileira de Climatologia, ano 8, v. 11, p. 87-103, 2012. DOI: http://dx.doi.org/10.5380/abclima.v11i0.28586 